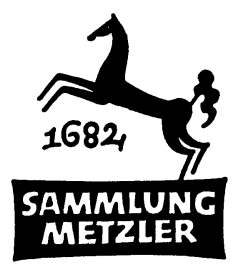

REALIEN ZUR LITERATUR

ABT. D:

LITERATURGESCHICHTE 


\author{
GOTTFRIED WEBER \\ UND \\ WERNER HOFFMANN
}

\title{
Gottfried
}

\section{von Straßburg}

5., von WERNER HOFFMANN bearbeitete Auflage

MCMLXXXI

J. B. METZLERSCHE VERLAGSBUCHHANDLUNG STUTTGART 
1.Aufl. (1.- 4.Tsd.) April 1962

2.Aufl. (5.- 8.Tsd.) April 1965

3. Aufl. (9.-12.Tsd.) Juni 1968

4. Aufl. (13.-16. Tsd.) Juni 1973

5. Aufl. (17.-21.Tsd.) September 1981

CIP-Kurztitelaufnahme der Deutschen Bibliothek

Weber, Gottfried:

Gottfried von Straßburg / Gottfried Weber u. Werner Hoffmann. - 5., von Werner Hoffmann bearb. Aufl. - Stuttgart: Metzler, 1981.

(Sammlung Metzler; M 15: Abt. D, Literaturgeschichte)

ISBN 978-3-476-15015-8

NE: Hoffmann, Werner; GT

ISBN 978-3-476-15015-8

ISBN 978-3-476-04141-8 (eBook)

DOI 10.1007/978-3-476-04141-8

M 15

(C) 1981 Springer-Verlag GmbH Deutschland

Ursprünglich erschienen bei J. B. Metzlersche Verlagsbuchhandlung und Carl Ernst Poeschel Verlag GmbH in Stuttgart 1981 


\section{VORWORT ZUR 5. AUFLAGE}

Wenn ein Dichter, über den wir kaum etwas wissen, und sein Werk, von dem nur wenige Fakten sicher sind, dessen Deutung aber in hohem Maße kontrovers ist, in einer auf die sRealien zielenden Darstellung präsentiert werden sollen, dann liegt es nahe, in dieser besonders die unterschiedlichen Forschungspositionen zu referieren und die strittigen Probleme zu kennzeichnen. So ist es in dem Bändchen über Gottfried von Straßburg von Anfang an geschehen, und ich habe, in Übereinstimmung mit Gottfried Weber, die stark forschungsgeschichtliche Orientierung auch in der neubearbeiteten 5. Auflage beibehalten und im Hinblick auf die neuere Forschung, ihre Ansätze und Ergebnisse wie ihre Problematik, sogar noch ausgedehnt. Die Aktualisierung erstreckt sich natürlich nicht zuletzt auf die Literaturangaben. Hans-Hugo Steinhoffs ausgezeichnete >Bibliographie zu Gottfried von Straßburg aus dem Jahre 1971 erlaubt es, die älteren Arbeiten in relativ straffer Auswahl zu verzeichnen; die jüngeren und namentlich die von Steinhoff nicht mehr erfaßten Beiträge zum >Tristan « aus den siebziger Jahren sind dagegen in einer sehr viel breiteren Auswahl aufgenommen. Sie alle für die Darstellung auszuwerten war allein schon aus Raumgründen unmöglich. Auch kam es mir mehr darauf an, eine Reihe von zentralen Aspekten des >Tristan « und seiner nach wie vor umstrittenen Deutung innerhalb des vorgegebenen Rahmens einigermaßen umfassend aufzuzeigen, als eine unvermeidlicherweise verwirrende Fülle von Einzelheiten auszubreiten und $\mathrm{zu}$ diskutieren.

Stellennachweisen und Zitaten aus Gottfrieds >Tristan $<$ liegt die Ausgabe Friedrich Rankes zugrunde. In den gleichfalls verbreiteten Ausgaben des >Tristan durch Karl Marold sowie Reinhold Bechstein/Peter Ganz ergeben sich geringfügige Abweichungen in der Verszählung gegenüber der Rankeschen Ausgabe: Von Vers 241 bis 5266 (nach Ranke) bleibt diejenige der beiden anderen Ausgaben um jeweils zwei Verse zurück, von Vers 5267 an ist sie in ihnen der Rankeschen Zählung um vier Verse voraus, da Ranke nach Vers 5266 seines Textes sechs Verse als unecht athetiert hat.

Mannheim, im Juni 1981

W. H. 


\section{INHALT}

I. Kapitel: Zur Person des Dichters ........... 1

II. Kapitel: Gottfrieds Dichtungen ............. 9

1. Die Gottfried zugeschriebenen lyrischen

Dichtungen................ 9

2. Die Überlieferung von Gottfrieds >Tristan<. 12

3. Zur Entstehung (Datierung) des >Tristan`. . 17

4. Zu Gottfrieds Sprach- und Verskunst. . . . 25

III. Kapitel: Der Tristanstoff in der mittelalterlichen

Dichtung................. 31

IV. Kapitel: Forschungsgeschichte, Forschungsstand,

Forschungsaufgaben ............ 58

Auswahlbibliographie .................... 102

Register... . . . . . . . . . . . . . . . . . . . . . 119 
Zeitschriften, Sammelwerke, wissenschaftliche Reiben:

\begin{tabular}{|c|c|}
\hline $\mathrm{AB} \ddot{\mathrm{A} G}$ & Amsterdamer Beiträge zur Älteren Germanistik \\
\hline $\mathrm{ADB}$ & Allgemeine Deutsche Biographie \\
\hline AfdA & Anzeiger für deutsches Altertum und deutsche Literatur \\
\hline Arch. & $\begin{array}{l}\text { Archiv für das Studium der neueren Sprachen und Lite- } \\
\text { raturen }\end{array}$ \\
\hline ATB & Altdeutsche Textbibliothek \\
\hline AUMLA & $\begin{array}{l}\text { Journal of the Australasian Universities Language and } \\
\text { Literature Association }\end{array}$ \\
\hline Beitr. & $\begin{array}{l}\text { Beiträge zur Geschichte der deutschen Sprache und Lite- } \\
\text { ratur }\end{array}$ \\
\hline CCM & Cahiers de Civilisation Médiévale \\
\hline $\mathrm{DPh}$ & $\begin{array}{l}\text { Deutsche Philologie im Aufriß, hg. von Wolfgang } \\
\text { Stammler }\end{array}$ \\
\hline DU & Der Deutschunterricht (Stuttgart) \\
\hline DVjs. & $\begin{array}{l}\text { Deutsche Vierteljahrsschrift für Literaturwissenschaft } \\
\text { und Geistesgeschichte }\end{array}$ \\
\hline Et. Germ. & Etudes Germaniques \\
\hline Euph. & Euphorion. Zeitschrift für Literaturgeschichte \\
\hline GAG & Göppinger Arbeiten zur Germanistik \\
\hline GLL & German Life and Letters \\
\hline GR & The Germanic Review \\
\hline GRM & Germanisch-Romanische Monatsschrift \\
\hline Hist. Jb. & Historisches Jahrbuch \\
\hline JbIG & Jahrbuch für Internationale Germanistik \\
\hline JEGPh & Journal of English and Germanic Philology \\
\hline
\end{tabular}

Kürschners DNL Deutsche National-Litteratur. Historisch kritische AusLeuv. Bijdr. Leuvense Bijdragen

LiLi LiLi. Zeitschrift für Literaturwissenschaft und Linguistik

MDU Monatshefte für deutschen Unterricht, deutsche Sprache und Literatur

Med. Aev. Medium Aevum

Mlat. Jb. Mittellateinisches Jahrbuch

MLQ Modern Language Quarterly

MLR The Modern Language Review

MSB Sitzungsberichte der Akademie der Wissenschaften zu

München. Philosophisch-philologische und historische Klasse

MTU Münchener Texte und Untersuchungen zur deutschen Literatur des Mittelalters

NDB Neue Deutsche Biographie 
NdJb.

Neophil.

PhStQ

PMLA

RG

RUB

SM

THSC

Triv.

UTB

VL

WB

WdF

WdF, Bd. 57 Wolfram von Eschenbach, hg. von Heinz Rupp, 1966 $(=\mathrm{WdF}, \mathrm{Bd} .57)$

WdF, Bd. 320 Gottfried von Straßburg, hg. von Alois Wolf, 1973 (= WdF, Bd. 320)

WW Wirkendes Wort

WZGreifswald Wissenschaftliche Zeitschrift der Ernst-Moritz-ArndtUniversität Greifswald. Gesellschafts- und Sprachwissenschaftliche Reihe

ZfdA Zeitschrift für deutsches Altertum und deutsche Literatur

ZfDk Zeitschrift für Deutschkunde

$\mathrm{ZfdPh} \quad$ Zeitschrift für deutsche Philologie

ZfG Zeitschrift für Germanistik

ZföG Zeitschrift für die österreichischen Gymnasien

$\mathrm{ZfrPh} \quad$ Zeitschrift für romanische Philologie

Andere Abkürzungen

$\begin{array}{llll}\text { Abh. } & \text { Abhandlung } & \text { Jg. } & \text { Jahrgang } \\ \text { Anm. } & \text { Anmerkung } & \text { Jh. } & \text { Jahrhundert } \\ \text { Aufl. } & \text { Auflage } & \text { NF } & \text { Neue Folge } \\ \text { Bd., Bde. } & \text { Band, Bände } & \text { o. J. } & \text { ohne Jahr } \\ \text { Diss. } & \text { Dissertation } & \text { S. } & \text { Seite } \\ \text { H. } & \text { Heft } & \text { Sp. } & \text { Spalte } \\ \text { hg. } & \text { herausgegeben } & \text { v. } & \text { Vers } \\ \text { Hs. } & \text { Handschrift } & & \end{array}$

